\title{
Application of Bio-inspired Algorithm of Structural Optimization to Automated Design
}

\author{
Mirosław Mrzygłód \\ Institute of Rail Vehicles, Cracow University of Technology, Al. Jana Pawla II 37, Cracow, Poland
}

\begin{abstract}
Keywords: Structural Optimization, Bio-inspired Algorithm, Automated Design.
Abstract: $\quad$ The article presents the concept of methodology of automated design based on bio-inspired algorithm of structural optimization. For the purposes of the automatic design, the constant criterion surface algorithm (CCSA) is used The algorithm shapes the structure under constant constraint surface rule and gives possibility to start optimization from a minimum volume arrangement. The automated design schema considers a minimum effort of the designer that is limited to defining the loads and boundary conditions. To ensure a high reliability of the automated design process, the CCSA algorithm was enriched by a procedure of the structure continuity control. As illustrated in the chair example, the application of bio-inspired algorithm in the automated design framework allows to obtain efficient solution.
\end{abstract}

\section{INTRODUCTION}

The high robustness of biological structures and their mechanism of auto-elimination of weak solutions can be taken as a direction of research on the modern design methodology. In order to obtain the optimum solutions fast it is necessary to eliminate the restrictions of human mind.

The article presents a concept of methodology of automated design based on bio-inspired algorithm of structural optimization. The assumption of such methodology is to reduce the contribution of the designer in the designing process only to defining loads and boundary conditions. For the purposes of the automatic design, the constant criterion surface algorithm (CCSA) will be used (Mrzyglod, 2012). The algorithm belongs to generative evolutionary design methods (Bentley, 1999) and use indirect representation similar to the cellular automaton (CA) algorithm (Tovar et al., 2006). However, the CA algorithm uses local rules to modify the structure.

The CCSA algorithm is based on the principles of constant stress surface rule. This idea was first formulated by Mattheck and Burkhardt (1990) and states that all biological structures follow the rule of a constant stress at the surface. Though, the condition of constant energy density at the free surface of the optimized structure was first derived by Wasiutyński (1960).
The CCSA algorithm gives the possibility to do optimization with various constraints as well as to solve multi-constraint problems. Moreover, it is also possible to start the optimization procedure from a minimum volume layout (unfeasible solution) and growth structure to its optimum topology. To ensure high reliability of the automated design process, the algorithm was enriched by a procedure of the structure continuity control. The procedure is based on the intelligent strategy of void identification (ISOVI) (Mrzyglod, 2011).

\section{THE CONSTANT CRITERION SURFACE ALGORITHM}

The optimization problem can be formulated as follows:

$$
\min _{\eta} f(\eta)
$$

the constraints are:

$$
g_{j}(x) \leq \overline{g_{j}}, j=[1,2, \ldots, K]
$$

where: $\eta=\left[\eta_{1}, \eta_{2}, \ldots \eta_{N}\right]$ is a vector of design variables defined as $\eta_{i}=E_{i} / E_{0}, E_{i}$ and $E_{0}$ are respectively, intermediate and real material Young's modules; $g_{j}(x)$ are the criterion parameters; $\overline{g_{j}}$ are 
the upper bounds of constraints. The $N$ design variables represent a pseudo-density (stiffness parameter) of each finite element of the structures that vary between $\eta_{\min }$ and 1 . The lower boundary of pseudo-density $\eta_{\min }$ is introduced to prevent singularity of the equilibrium problem.

The CSSA algorithm consists of procedure of the removal and adding procedure of FE elements (see Fig. 1). The removal procedure of FE elements with low values of constraint criterion parameters $g$ is similar to Evolutionary Structural Optimization (ESO) approach introduced by Xie and Steven (1993). However, in the CSSA algorithm the volume value of optimized structure is not assumed a priori what makes an important difference between those algorithms.

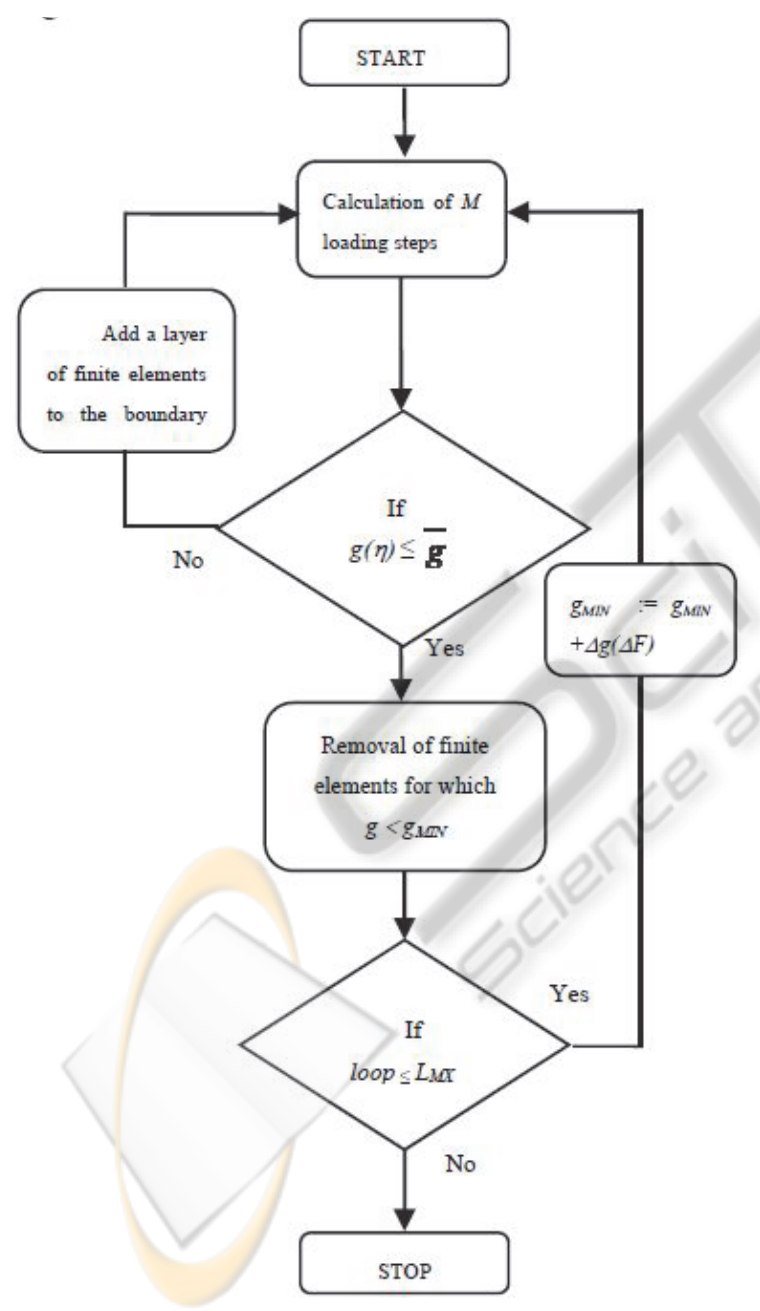

Figure 1: The constant criterion surface algorithm.

The elimination procedure is controlled by a $\Delta F$ parameter of volume percentage reduction. To select the constant value of $\Delta F$ (usually $1 \%$ ), a constraint criterion increasing parameter $\Delta g$ at every volume decreasing iteration is calculated. The FE elements with values of constraint criterion parameters $g$ below the $g_{M I N}$ limit are eliminated from the structure. For the volume increasing iteration, there is no removal operation.

The stress-constrained topology optimization procedure can give premature results when it is stuck in point of high values of the state parameter.

In the CCSA algorithm when criterion function is over the limit, a layer of finite elements is added to the entire boundary of the structure (see Fig. 1,2).

(a)

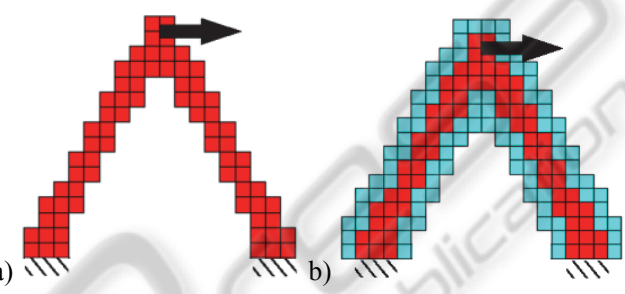

Figure 2: The layer expansion algorithm: a structure before (a) and after operation of adding a layer of finite elements to the structure boundary (b).

The procedure of increasing the volume of the structure is continued until the criterion parameter $g$ returns to admissible values. By increasing and decreasing the structure volume, the algorithm obtains better solutions after every 'breakdown'. This scheme is analogous to the simulated annealing (SA) (Kirkpatrick et al., 1983).

Furthermore, to ensure reliability of the optimization process, the continuity control procedure has been added to the CCSA algorithm. It prevents the optimization process from being stopped because of FE solver error. For the discontinuities detection, the ISOVI procedure based on automatic pattern recognition is employed (Mrzyglod, 2011). When the ISOVI procedure detects a loss of structural continuity, the layer expansion procedure is turned on. This procedure will work until the structure returns to the continuity. This can be treated as self-healing scheme of the optimization procedure.

The CCSA method to take into account forces that act in an asynchronous way on the structure, a 'compare and save maximum' procedure of summation of constrain criterion values is proposed (Mrzyglod, 2010). The procedure assumes, that during each iteration for every finite element only the maximum values of the constraint criterion of all load cases will be written to the equivalent design space. 
The constraint values of final equivalent design space are used by the constant criterion surface algorithm of topology optimization.

For multi-constrained topology optimization problems normalized constraints are introduced (Mrzyglod, 2012).

To test the convergence of the algorithm, the benchmark problem of optimizing truss topology was selected (Rozvany, 1998) (see Fig. 3a-b). In the Fig. $3 c-d$ the result of the tests are presented. The obtained numerical solution is consistent with data published in the literature for the compliance and stress constraints (Bendsoe and Sigmund, 2003).

From the example, we can see clear dependency of final solution on applied load values (see Fig. 3cd).

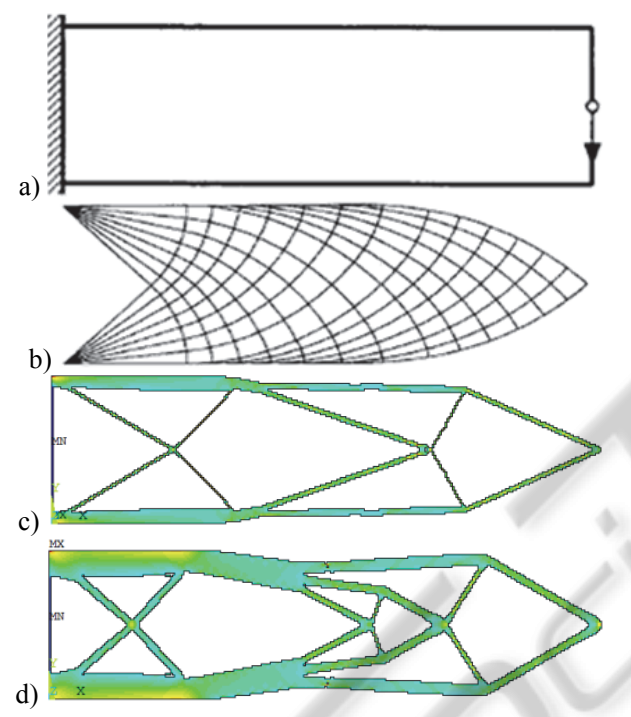

Figure 3: Benchmark problem (Rozvany, 1998): problem description (a), analytical solution (b), numerical solution for load Q (c), numerical solution for load $2 \times \mathrm{Q}$ (d).

\section{EXAMPLE OF AUTOMATED DESIGN}

The chair structure was selected as the example of automated design. In the example, the mass of the structure is optimized with the von Mises stress constraint ( $\bar{g}=0.5 \mathrm{MPa}, j=1$ ).

The structure is subjected to complex and asynchronous loading schema: a vertical force $\mathrm{Q}_{\mathrm{v}}$ that is acting on the seat of the chair; horizontal forces $Q_{h}=1 / 3 Q_{v}$ that are acting in four directions on the seat; a superposition of forces $\mathrm{Q}_{\mathrm{vb}}+\mathrm{Q}_{\mathrm{hb}}$ that are acting on the back of the chair $\left(Q_{\mathrm{vb}}=\mathrm{Q}_{\mathrm{hb}}=1 / 4\right.$ $Q_{v}$ ) (see Fig. 4a). The chair FE model with boundary and results of automated design procedure of the chair example are presented in Fig. 4a-f. The history of searching for a solution is shown in the Fig. 5.

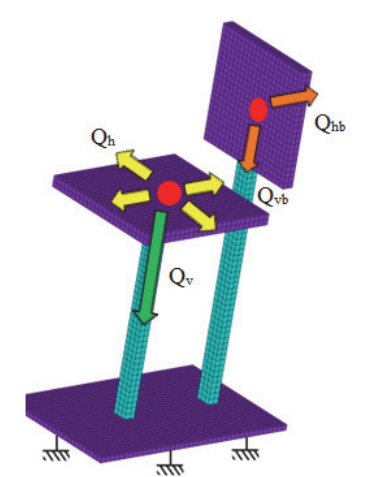

a)

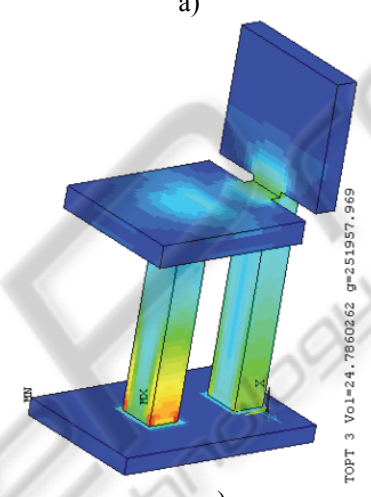

c)

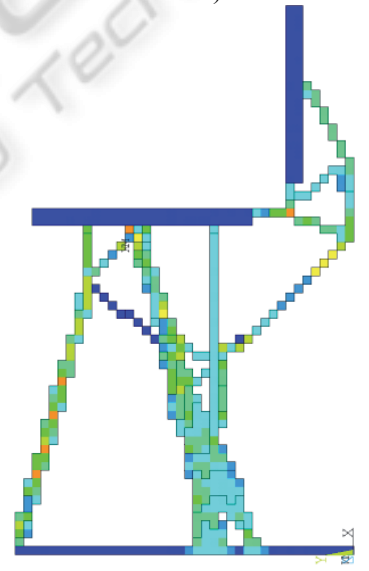

e)

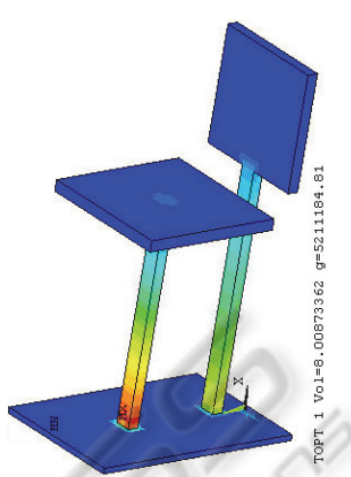

b)

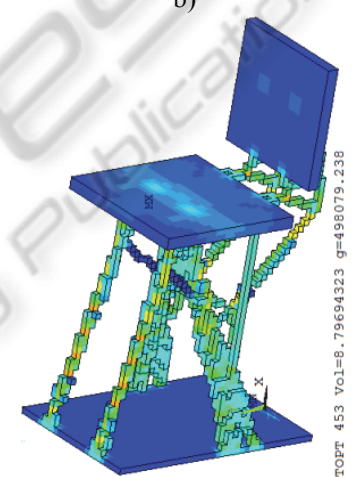

d)

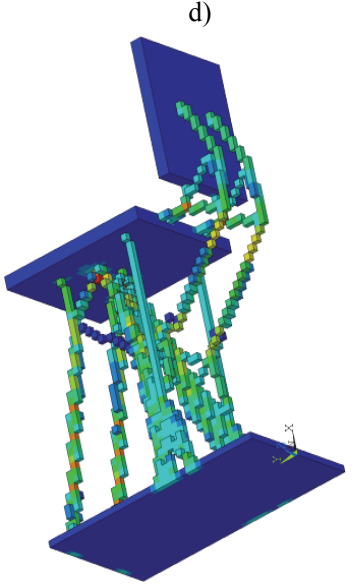

f)
Figure 4: Example of automated design: FE model with boundary conditions (a); starting solution (unfeasible) $\mathrm{Vol}=8.0 \%$ ( $\mathrm{Vol}$ - percent of volume of the design space $N$ $=64400)(\mathrm{b})$; first feasible solution $\mathrm{Vol}=24.8 \%$ (c) best solution $\mathrm{Vol}=8.8 \%(\mathrm{~d}, \mathrm{e}, \mathrm{f})$.

\section{CONCLUSIONS}

In the article, the design methodology based on bio- 
inspired algorithm is presented. In the algorithm CCSA, the bio-inspired procedure of shaping the structure in the form of constant criterion surface has been joined with local minima avoidance scheme based on simulated annealing. This ensure achieving the high efficiency of optimal solution searching. With the introduction of additional structure continuity control, the optimization procedure has been enriched by the possibility of self-repair which significantly increases the reliability of the optimization process. As illustrated in the example, the application of the automated design framework allowed to obtain efficient solution with minimum effort of the designer.

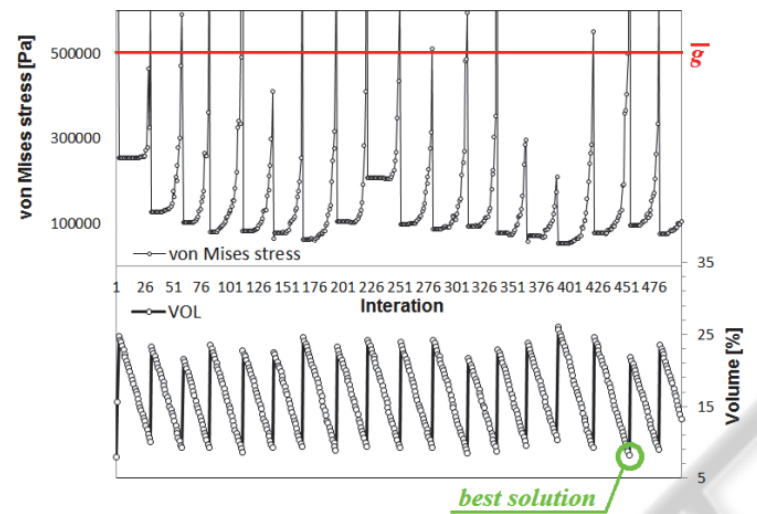

Figure 5: Example of automated design: History of searching for a solution $\left(L_{\max }=500\right)$.

\section{REFERENCES}

Bentley P. J. (Ed.), 1999. Evolutionary design by computers, Morgan Kaufmann Publishers, San Francisco, CA.

Bendsoe M. P., Sigmund O., 2003. Topology Optimization. Theory, Methods, and Applications, Springer Verlag, New York.

Mattheck C., Burkhardt S., 1990. A new method of structural shape optimisation based on biological growth, Int. J. Fatigue, 12(3), 185-190.

Mrzyglod, M., 2010. Two-stage optimization method with fatigue constraints for thin-walled structures, Journal of Theoretical and Applied Mechanics, 48(3), 567578.

Mrzyglod, M., 2011. A Method of Voids Size Identification for $2 \mathrm{D}$ and $3 \mathrm{D}$ Topology. The 2 nd International Conference on Inverse Problems in Mechanics, IPM2011 ECCOMAS Thematic Conference, Rzeszow University of Technology, 7374.

Mrzyglod M., 2012. Multi-constrained topology optimization using constant criterion surface algorithm, Bulletin of the Polish Academy of Sciences
- Technical Sciences, 60(2), 229-236.

Kirkpatrick S., Gelatt C.D., Vecchi M. P., 1983. Optimization by Simulated Annealing, Science, 220, 671-680.

Rozvany G. I. N., 1998. Exact analytical solutions for some popular benchmark problems in topology optimization, Struct Optim 15, 42-48.

Tovar A., Patel N. M., Niebur G. L., Sen M., Renaud J. E., 2006. Topology Optimization Using a Hybrid Cellular Automaton Method with Local Control Rules, ASME Journal of Mechanical Design, 128(6), 1205-1216.

Wasiutyński Z., 1960. On the congruency of the forming according to the minimum potential energy with that according to equal strength, Bull. De L'Academie Polonaise des Sciences, Serie des Sciences Techniques, 8(6), 259-268.

Xie Y. M., Steven G. P., 1993. A simple evolutionary procedure for structural optimization, Comput. Struct., $49,885-896$. 